Editorial

\title{
Acknowledgement to Reviewers of JPM in 2018
}

\author{
JPM Editorial Office \\ MDPI, St. Alban-Anlage 66, 4052 Basel, Switzerland \\ Published: 17 January 2019
}

\begin{abstract}
Rigorous peer-review is the corner-stone of high-quality academic publishing. The editorial team greatly appreciates the reviewers who contributed their knowledge and expertise to the journal's editorial process over the past 12 months. In 2018, a total of 38 papers were published in the journal, with a median time to first decision of 27.5 days and a median time to publication of 65.5 days. The editors would like to express their sincere gratitude to the following reviewers for their cooperation and dedication in 2018:
\end{abstract}
Alaimo, Alessandro
Gouin-Thibault, Isabelle
Alfirevic, Ana
Goyenvalle, Aurélie
Antoñanzas, Fernando
Graber, Joel
Bien, Stephanie A.
Hagenbuch, Bruno
Boisvert, François-Michel
Hart, Steven
Bonham, Vence L.
Herraez, Elisa
Burns, Katie
Hiratsuka, Masahiro
Butler-browne, Gillian
Hizel, Candan
Castel, Victoria
Hoppu, Ulla
Cheng, $\mathrm{Xi}$
Hult, Kristopher J.
Chmurzynska, Agata
Hvas, Anne-Mette
Čipak Gašparović, Ana
Inamura, Kentaro
Clementi, Emilio
Janowski, Miroslaw
Codacci-Pisanelli, Giovanni
Jirák, Daniel
Cohen, Stephanie
Karnes, Jason H.
Crona, Daniel
Kato, Yukio
Defesche, Joep C.
Kulak, Wojciech
DeLeon, Jose
Lanfear, David
DeWald, Tracy
Lazzeri, Chiara
Dijkstra, Jouke
Lewis, Gladius
Du Preez, Ilse
$\mathrm{Li}$, Yvonne
Engvall, Jan
López Fernández, Luis
Ewing, Adam
Gagliardi, Paolo Armando
Maeda, Hiroshi
Gallegos, Martha Patricia
Mather, Carey
Garrison, Louis
Meng, Jinhong
Gibson, Greg
Mills, Rachel
Glueck, Charles J.
Mitchell, Cassie S.
Gong, Yan
Moyle, Louise
Naj, Adam 
Navab, Mohamad

Nigam, Sanjay

O'Rourke, Patricia Pearl

Pan, Minggui

Patch, Christine

Perera, Minoli

Perez, Laurent

Pergament, Eugene

Persson, Cecilia

Pfau, Steven E.

Pyles, Lee A.

Radhakrishnan, Kavita

Ragia, Georgia

Reynolds, Jessica L.

Sanderson, Saskia

Sandman, Lars

Savoia, Carmine

Scott, Erick R

Sharfaei, Sadaf

Sharma, Atul

Sharma, Naveen

Shipman, Kate

Silva, Susana N.
Singh, Sonal

Singla, Bhupesh

Skinner, Debra

Stewart, Alexandre

Taylor, Casey

Toffoli, Giuseppe

Tolstikov, Vladimir

Tougeron, David

Vafiadaki, Elizabeth

Van Putten, Maaike

Van Schaik, Ron H.N.

Vickers, Kasey C.

Villa, Chiara

Vimaleswaran, Karani S.

Wang, Xinwen

Welch, Carrie

Williams, Janet $\mathrm{K}$.

Wilton, Steve

Wojtkiewicz, Joanna

Wu, Alan

Zaiss, Dietmar M.W.

Zhou, Jingying

Ziegelstein, Roy

(C) 2019 by the authors. Licensee MDPI, Basel, Switzerland. This article is an open access article distributed under the terms and conditions of the Creative Commons Attribution (CC BY) license (http://creativecommons.org/licenses/by/4.0/). 